\title{
Pobreza multidimensional y vulnerabilidad social
}

\author{
Eramis Bueno Sánchez
}

Como fenómeno de larga data, la pobreza ha sido abordada desde múltiples definiciones, enfoques y métodos de medición. Ya sea que consideremos a la pobreza como falta de ingresos, necesidades básicas insatisfechas o vulneración de capacidades, o que asumamos la visión subjetiva de quienes la padecen, es inevitable concluir que se trata de uno de los problemas más graves de la humanidad, de no cambiarse las reglas de los modelos sociales. Estimulados inicialmente por Carolina E. Moser, muchos cientistas sociales han sido atraídos al estudio de la vulnerabilidad social. Ambos ejes de la relaciones entre población y desarrollo serán abordados aquí, haciendo referencia al caso de México. 
ENFOQUE MULTIDIMENSIONAL DE POBREZA.

INCORPORACIÓN DE LA VULNERABILIDAD SOCIAL

La pobreza degrada y destruye, moral, social y biológicamente al más grande milagro cósmico: la vida humana. La existencia de la pobreza es una aberración de la vida social, un signo evidente del mal funcionamiento de la sociedad. JULIO BoltVINIK

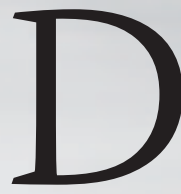
esde la primera década del presente siglo se ha venido formulando el enfoque multidimensional de la pobreza, que quedó plasmado en el Informe sobre el Desarrollo Mundial de 2001 publicado por el Banco Mundial (BM). En el Informe se mide la pobreza en fun- 
ción de tres aspectos: ${ }^{1}$ «la oportunidad refleja el ingreso individual, el consumo y el nivel de desigualdad de una sociedad»; «la potenciación refleja la participación individual en la adopción de decisiones y queda fortalecida por la descentralización, la transparencia y la obligación de rendir cuentas en todos los aspectos de la gobernabilidad, incluido el ordenamiento de los recursos naturales», y «la seguridad refleja el grado de protección individual contra sacudidas económicas y violencia personal». Además, se considera que «las capacidades reflejan el nivel de alfabetización y de salud del individuo».

El enfoque multidimensional de pobreza ha sido desarrollado, con diferentes matices, por organismos internacionales ${ }^{2}$ y se ha plasmado en diversos estudios nacionales. ${ }^{3}$ El Informe sobre el Desarrollo Humano $2010^{4}$ presenta el Índice de Pobreza Multidimensional, que complementa a los índices basados en medidas monetarias y considera las privaciones que experimentan las personas pobres y el marco en que éstas ocurren. El índice identifica una serie de privaciones en las mismas tres dimensiones del Índice de Desarrollo Humano (IDH) y muestra el número de personas que son pobres (que sufren privaciones) y el número de privaciones con las que usualmente vive una familia pobre. La multidimensionalidad de la pobreza es referida a las aportaciones de Amartya Sen. ${ }^{5}$

El concepto de pobreza alude a dimensiones como la económica, cultural y espiritual. No obstante, lo económico suele ser determinante e interactúa con otros factores. En ese tenor, la pobreza no sólo se refiere a la carencia de recursos monetarios para acceder a bienes y servicios, sino también a un conjunto de factores como la dificultad para ganarse la vida, dependencia, falta de poder y voz, ignorancia, desempleo, enfermedad, tristeza, humildad, desnutrición, mendicidad, angustia, falta de oportunidades, pereza y conformismo. La pobreza se vincula a todas estas carencias sociales e individuales (educación, salud, trabajo) que tienen relación con la vulnerabilidad y susceptibilidad de los pobres ante los riesgos.

Al tiempo que se avanza en el desarrollo e implementación del enfoque multidimensional, ha ido ganando espacio, desde el punto de vista conceptual y metodológico, la identificación de situaciones de vulnerabilidad social que acompañan o complementan el tratamiento de la problemática de la pobreza. La identificación de situaciones de vulnerabilidad responde a una nueva mirada sobre los problemas sociales. Esta mirada no se concentra en la situación consumada de pobre$\mathrm{za}$, sino que pretende poner en evidencia circunstancias más complejas, que si bien se acercan al estado de pobreza, no necesariamente se detectan con los métodos de medición desarrollados en la mayor parte de los trabajos publicados en la literatura especializada.

La revisión de la literatura permite constatar que la construcción de modelos teórico-metodológicos basados en la noción de vulnerabilidad está contribuyendo a la interpretación de la pobreza y desigualdad persistentes en América Latina. A partir de los trabajos de Moser, se produjo un enfoque de vulnerabilidad centrado en los grupos vulnerables, para orientar el análisis a los activos y desventajas sociales. Simultáneamente, la Comisión Económica para América Latina y el Caribe (CEPAL) puso énfasis en las vulnerabilidades social, demográfica y ambiental, aplicando un enfoque teórico-metodológico para examinar determinadas regiones y países, o bien a grupos específicos como la juventud y personas de la tercera edad.

No es nuevo decir que el complejo y multifacético problema de la pobreza guarda estrecha relación con el tema emergente de la vulnerabilidad social. Los grupos pobres sufren intensamente una mayor vulnerabilidad social, a la que están expuestos la mayoría de los hogares de América Latina y, en relación con esto, se asume que el enfoque de la pobreza es insuficiente para comprender las complejas situaciones que aparecen en el contexto del patrón de desarrollo consolidado en las últimas décadas en los países de la región.

El asset/vulnerability framework planteado por Moser $^{6}$ se centra en las relaciones entre pobreza, características de los hogares y su entorno. Según esta concepción, los pobres enfrentan la precariedad al recurrir a activos tangibles (trabajo, capital humano, ${ }^{7}$ vivienda) o intangibles (relaciones domésticas y capital social); ${ }^{8}$ además, resalta el papel de los activos de los pobres, no el de sus pasivos, lo que sugiere que las políticas apropiadas para salir de la pobreza y enfrentar las crisis socioeconómicas deben promover el uso de los primeros. Cabe destacar que más que proponer una definición de activos, Moser define categorías para los activos de las personas (mujeres, hombres y niños), hogares y comunidades pobres de las zonas urbanas en función de un quíntuple «marco de vulnerabilidad de los activos».

John Toye plantea que si se mira a los pobres como agentes, y no como víctimas, se requeriría conocer mucho más sobre cómo actúan y qué hacen en las diversas situaciones de vulnerabilidad y pobreza. ${ }^{9}$

El énfasis que se pone en reconocer la relevancia de los activos surge del cuestionamiento que se hace a 
muchas intervenciones dirigidas a combatir la pobreza y que actúan esencialmente sobre los ingresos, despreocupándose de los mecanismos, factores, características y oportunidades que tienen, adquieren o reciben los actores para consolidar su inserción socioeconómica.

Attanasio y Székely apuntan que los activos relevantes para los pobres son aquéllos que "permiten generar ingresos» y que son una función de la combinación de cuatro elementos decisivos: 1) el acervo de activos generadores de ingresos que posee una persona; 2) la tasa a la que se utilizan dichos activos para producir ingresos; 3) el valor de mercado de los activos generadores de ingresos, y 4) las transferencias y legados independientes de los activos generadores de ingresos poseídos. ${ }^{10}$

Debido a cómo es entendida la vulnerabilidad en los trabajos de Moser, Attanasio y Székely, se desprende que la mayor debilidad objetiva de los pobres, para enfrentar su supervivencia cotidiana y los efectos de las crisis económicas, podría ser contrarrestada con una administración de los activos disponibles, independientemente de lo escaso del ingreso. Con sólo considerar esto, resulta ya evidente que se apuesta a un cambio radical en los presupuestos que asumen las políticas dirigidas a superar la pobreza, desplazando el énfasis en la carencia de ingresos hacia la necesidad de contribuir a una apropiada dotación y movilización de todos los recursos (activos) y capacidades.

Luego de los trabajos iniciales de Moser, los desarrollos analíticos más sistemáticos sobre vulnerabilidad social en América Latina pueden encontrarse en los trabajos de Rubén Kaztman sobre Argentina y Uruguay, y más ampliamente en la labor realizada en el contexto de la CEPAL. Kaztman ha puesto de manifiesto que los recursos que controlan los hogares no se pueden valorar con independencia de la estructura de oportunidades a la que tienen acceso, esto es, los recursos se convierten en activos en la medida que permiten el aprovechamiento de las oportunidades que ofrece el medio a través del mercado, el Estado o la sociedad. Afirma que las estructuras de oportunidades no son una constante sino una variable, lo que quiere decir que los países no son iguales en materia de oportunidades, como tampoco lo son los diferentes momentos históricos ni su trayectoria.

De lo anterior se desprende que:

1) el nivel de vulnerabilidad de un hogar - que se refiere a su capacidad para controlar las fuerzas que lo afectan- depende de la posesión o control de activos, esto es, de los recursos requeridos para el aprovechamiento de las oportunidades que brinda el medio en que se desenvuelve, y 2) los cambios en la vulnerabilidad de los hogares pueden producirse por cambios en los recursos que posee o controla, por cambios en los requerimientos de acceso a la estructura de oportunidades de su medio o por cambios en ambas dimensiones. Un caso particular, pero muy frecuente, es un cambio asincrónico donde los requerimientos de acceso a las nuevas estructuras de oportunidades se modifican a mayor velocidad que la que utilizan los hogares para generar los recursos para su aprovechamiento. ${ }^{11}$

Las estructuras de oportunidades son definidas por Kaztman como probabilidades de acceso a bienes y servicios o al desempeño de actividades. Estas oportunidades inciden sobre el bienestar de los hogares, ya sea porque permiten o facilitan a los miembros del hogar el uso de sus propios recursos o porque les proveen recursos nuevos. ${ }^{12}$

Mientras Moser distingue entre trabajo, capital humano (salud y educación), activos productivos (vivienda), relaciones del hogar y capital como tal, Kaztman se refiere a capital financiero, capital físico, capital humano (incluyendo trabajo) y capital social. ${ }^{13}$

\section{POBREZA MULTIDIMENSIONAL Y Vulnerabilidad social en MÉxico}

El Consejo Nacional de Evaluación de la Política de Desarrollo Social (CONEVAL) había utilizado un modelo para medir la pobreza basado en el ingreso mensual per cápita requerido para cubrir, en términos de una canasta básica, las necesidades asociadas a tres líneas de pobreza: 1) alimentaria: incapacidad para adquirir una canasta básica alimentaria (conocida como la medida de pobreza extrema); 2) de capacidades: insuficiencia de ingresos para efectuar los gastos necesarios en salud y educación, y 3) de patrimonio: insolvencia para los gastos de vivienda, vestido y transporte (situación general de pobreza). Con este enfoque, después de un descenso sostenido en el periodo 1996-2006, los tres tipos de pobreza se habrían incrementado significativamente a partir de 2006 (véase gráfico 1).

Este modelo estaría siendo sustituido por una noción de pobreza multidimensional que la considera como un fenómeno social donde las personas poseen características cuantitativas y cualitativas más allá de la 
dimensión monetaria. La definición considera que una persona se encuentra en condiciones de pobreza multidimensional «si no tiene garantizado el ejercicio de al menos uno de sus derechos para el desarrollo social, y si sus ingresos son insuficientes para adquirir los bienes y servicios que requiere para satisfacer sus necesidades». ${ }^{14}$ La nueva propuesta reconoce que los derechos fundamentales son la expresión de necesidades, valores, intereses y bienes que deben ser considerados como esenciales y comunes a los seres humanos. ${ }^{15}$ Debido a que los derechos humanos son universales, inherentes, indivisibles e interdependientes, los avances o retrocesos asociados a la pobreza dependerán del cumplimiento de todos los derechos en su conjunto y no sólo de algunos de ellos: «la falta o agravio de uno afecta la integridad de las personas». ${ }^{16}$

GRÁFICO 1

México: evolución de la pobreza por ingresos, periodo 1992-2010

(porcentaje de personas)

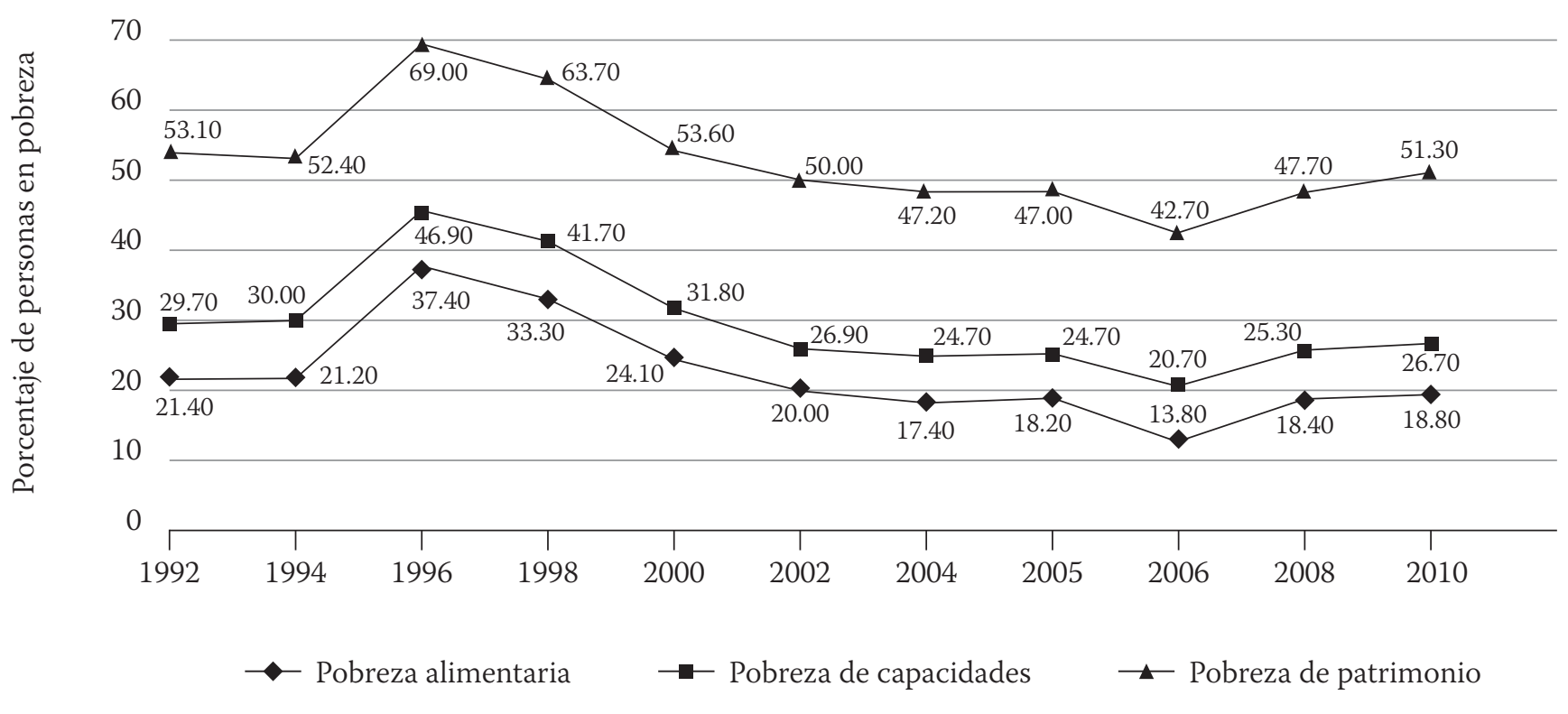

Fuente: estimaciones de CONEVAL con base en las ENIGH de 1992 a 2010.

El bienestar económico, medido a través del ingreso, permite conocer las necesidades que pueden ser satisfechas mediante la adquisición de bienes y servicios que se obtienen en los mercados con recursos monetarios. Uno de los objetivos de este espacio es identificar las condiciones económicas que limitan la libertad de las personas para desarrollarse plenamente. ${ }^{17}$

Un tercer espacio analítico calificado como «contexto territorial» se refiere a que la medición de la pobreza debe incluir el grado de cohesión social. Sin embargo, se reconoce que este indicador no constituye una cualidad de los individuos o de los hogares en pobreza sino de los contextos sociales (ciudades, barrios, pueblos o comunidades) en los que viven. Si bien el grado de cohesión social no juega el mismo papel que las carencias de ingreso o de acceso al cumplimiento de los derechos sociales, si proporciona el conjunto de relaciones que ofrecen posibilidades y recursos a las personas y a los hogares para enfrentar sus carencias. «Dada la naturaleza relacional y comunitaria de la cohesión social, su tratamiento metodológico y estadístico es distinto al de las carencias económicas y sociales». ${ }^{18}$

Dentro de este horizonte conceptual, la manera cómo la pobreza y la vulnerabilidad son entendidas y desarrolladas conlleva a que para definir y medir estos fenómenos la construcción propuesta considere tres espacios analíticos: derechos sociales, bienestar económico y contexto territorial. ${ }^{19}$ De particular interés para nuestro 
análisis es que esta nueva propuesta incorpora explícitamente la cuestión de la vulnerabilidad, identificando cuatro grupos poblacionales de interés para la política social: $i$ ) pobres multidimensionales, ii) personas vulnerables por carencia social, iii) personas vulnerables por ingreso y $i v$ ) personas que no tienen carencias sociales ni de ingreso. ${ }^{20}$ La población pobre multidimensional es aquélla que presenta carencias en los espacios i) y ii). La población vulnerable por carencias sociales es aquélla que, a pesar de tener un ingreso superior a la línea de bienestar económico (LBE) ${ }^{21}$ su índice de privación es mayor a uno, es decir, tiene una o más carencias sociales. La población vulnerable por ingresos es la que tiene un ingreso menor de la LBE y que no tiene carencias sociales. La población sin carencias sociales y con un ingreso superior a la LBE son quienes no son pobres multidimensionales ni vulnerables. ${ }^{22}$

A partir de la metodología de cálculo de la pobreza y vulnerabilidad asociada a este nuevo modelo, se observa, para los dos años en que se ha aplicado la propuesta, una mejoría en algunos indicadores, al tiempo que el monto de población en condiciones de pobreza y vulnerable por ingreso se habría incrementado considerablemente para un periodo corto, como el comprendido entre 2008 y 2010 (véase gráfico 2). Ni que decir que el 21.8\% de la población de México es no pobre y no vulnerable, esto es, $78.2 \%$ experimenta algún tipo de pobreza.

\section{Palabras Finales}

Los argumentos, descubrimientos y lecciones relativos a la pobreza multidimensional y la vulnerabilidad social son relevantes para enriquecer el arsenal teórico-metodológico disponible para el abordaje de estos trascendentales problemas sociales. Entre tanto, tratándose de problemáticas que dicen tener relación con muchos de los factores que integran la agenda de población y desarrollo, se hace más que evidente la conveniencia de analizarlas en el contexto de los ejes o nudos articuladores de tales relaciones.

\section{GRÁFICO 2}

México, pobreza multidimensional y población vulnerable.

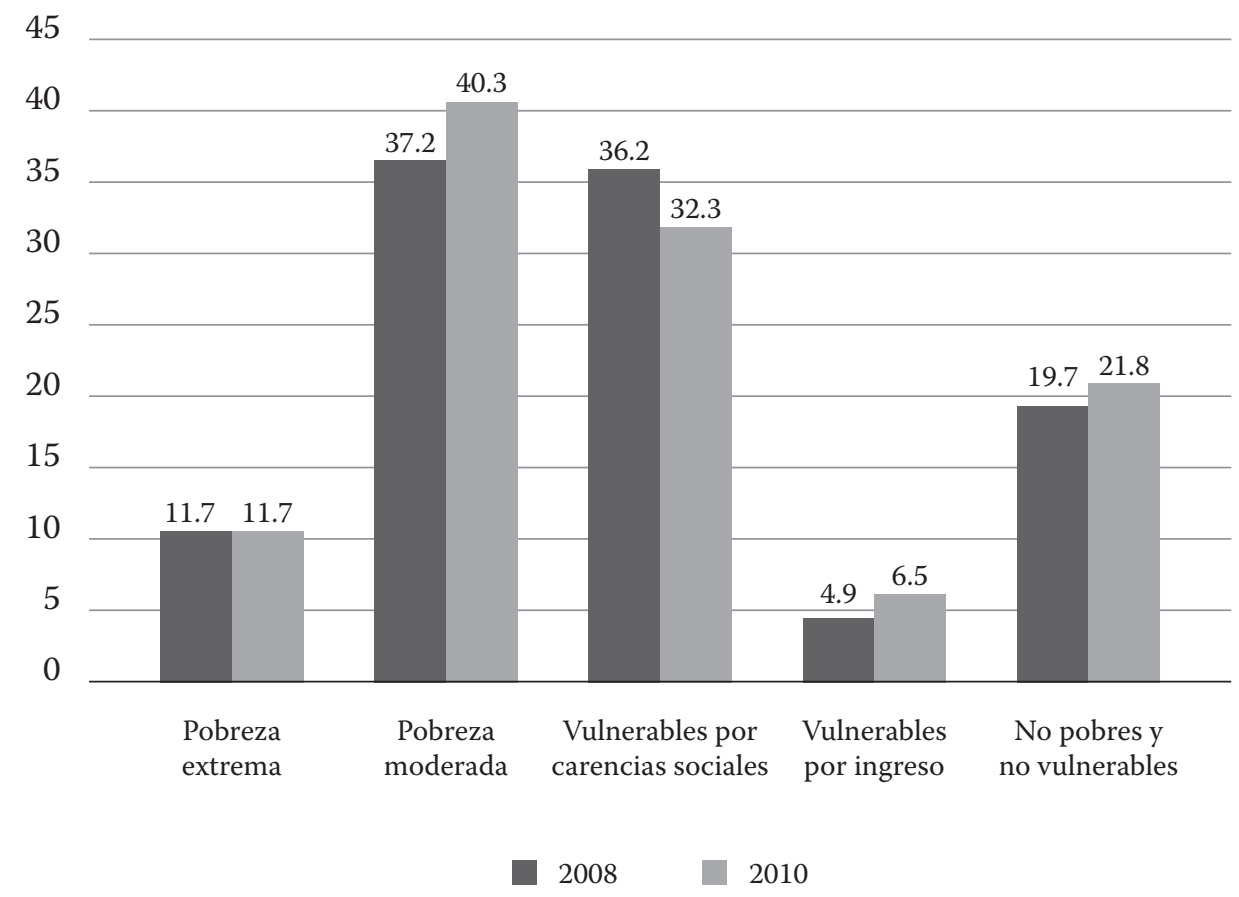

Fuente: Coneval, Pobreza en México y en las entidades federativas 2008-2010. 
1 UnfPa, Estado de la Población Mundial 2001. Nueva York, 2001, p. 30.

2 En julio de 2010, la Iniciativa de Oxford sobre la Pobreza y el Desarrollo Humano (OPHI) de la Universidad de Oxford presentó junto con la Oficina del Informe sobre Desarrollo Humano del Programa de las Naciones Unidas para el Desarrollo (PNUD) una nueva forma de medir la pobreza, que plantea una visión «multidimensional» de las personas que viven en la pobreza y que, según sus creadores, podría ayudar a asignar recursos de desarrollo de forma más efectiva. El IPM sustituye al Índice de Pobreza Humana, que ha venido formando parte de los Informes sobre Desarrollo Humano anuales desde 1997. Los resultados aportados por el Índice de Pobreza Multidimensional se dieron a conocer en el foro político de Londres y en línea en el sitio web de la OPHI.

3 Rodrigo Arim y Andrea Vigorito, Un análisis de la pobreza multidimensional en Uruguay 1991-2005, Serie Documentos de Trabajo DT 10/06, Uruguay, Instituto de Economía, marzo, 2007.

4 PNUD, Informe sobre el desarrollo humano 2010. La verdadera riqueza de las naciones: Caminos al desarrollo humano, 2010, p. 105.

5 Julio Boltvinik, «Conceptos y medición de la pobreza. La necesidad de ampliar la mirada», Papeles de Población, número 38, octubre-diciembre, 2003, pp. 9-25.

6 Caroline Moser, «Reassessing urban poverty reduction strategies: The asset vulnerability framework», World development, volumen 26, número 1, enero, 1998, pp. 1-19.

7 La mayor parte del capital humano se forma con la educación o la capacitación, que incrementan la productividad económica de una persona, es decir, le permiten obtener ingresos más altos. Los gobiernos, los trabajadores y los empleadores invierten en capital humano dedicando dinero y tiempo a la educación y la capacitación (acumulación de conocimientos y aptitudes). Como toda otra inversión, la inversión en capital humano exige sacrificios. La gente está de acuerdo en realizarlos si considera que, a cambio, va a obtener mayores ingresos en el futuro (Banco Mundial).

8 Caroline Moser, op. cit., p. 21.

9 Julio Boltvinik y Araceli Damian, La pobreza en México y el mundo. Realidades y desafíos, México, Siglo xxi, 2005, p. 85.

10 Orazio Attanasio y Miguel Székely, «La pobreza en América Latina. Análisis basado en los activos», El Trimestre Económico, número 263, julio-septiembre, 1999, p. 321.

11 Rubén Kaztman (coordinador), Activos y estructuras de oportunidades. Estudios sobre las raíces de la vulnerabilidad en Uruguay, Uruguay, CEPAL, junio, 1999, p. 20.
12 Ibidem, p. 21.

13 Ibidem, pp. 329-330.

14 CONEVAL, Informe de pobreza multidimensional en México, 2008, México, 2010, p. 26.

15 Ibidem, p. 25.

16 Ibidem, p. 26

17 Ibidem, p. 26

18 Ibidem, pp. 25-26.

19 Ibidem, p. 24.

20 Ibidem, pp. 12-13.

21 LBE mide el potencial del ingreso para satisfacer la totalidad de necesidades alimentarias y no alimentarias de las personas.

21 CONEVAL, Informe de pobreza multidimensional en México, 2008, p. 28. 


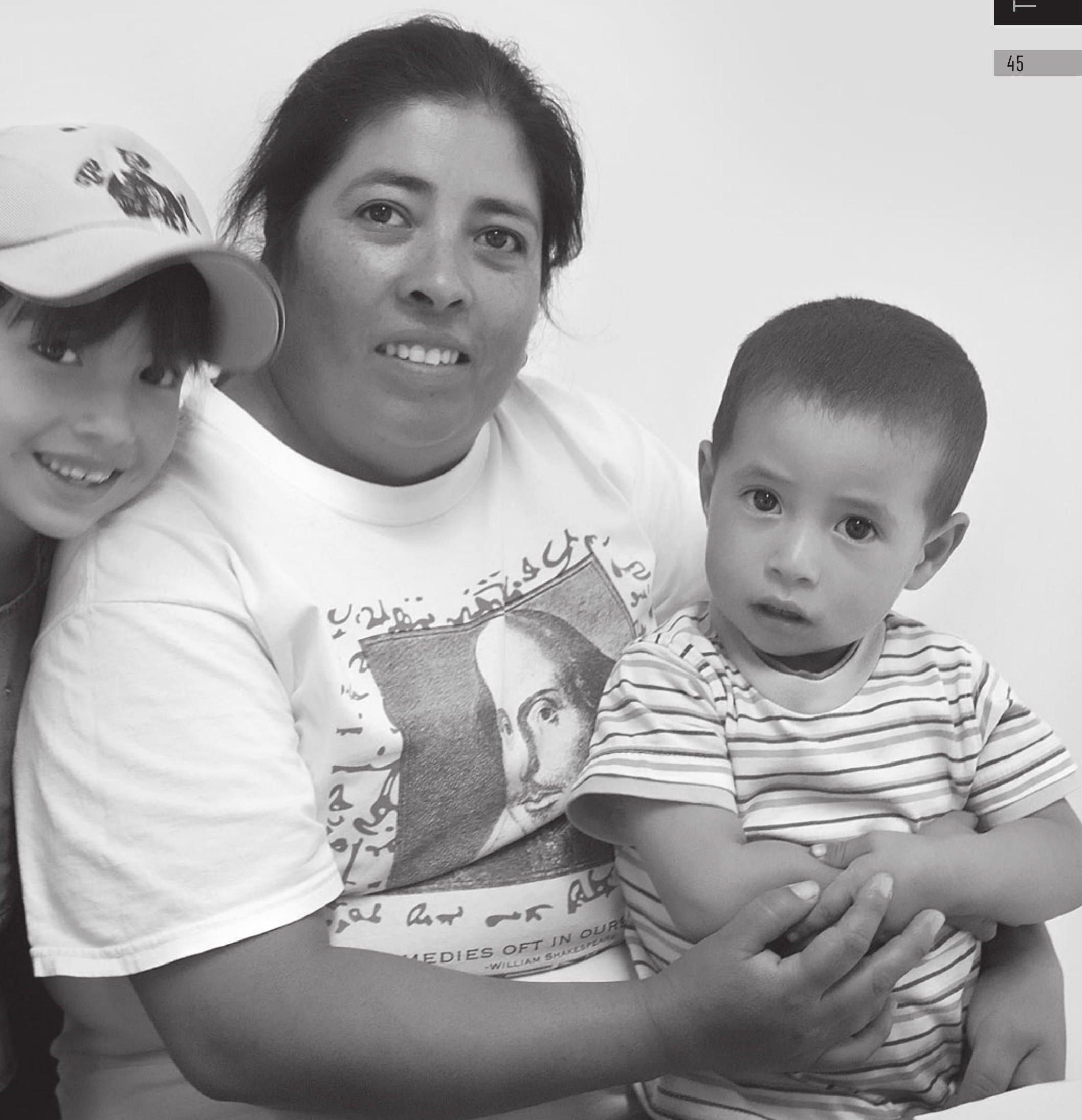

